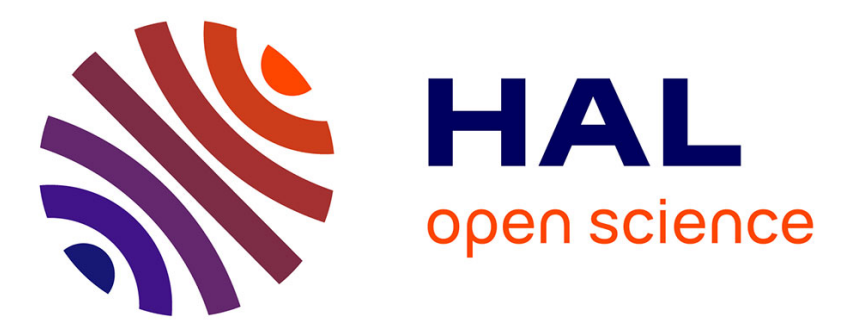

\title{
Applying the Mahalanobis-Taguchi System to Vehicle Handling
}

Elizabeth A. Cudney, Kioumars Paryani, Kenneth M. Ragsdell

\section{To cite this version:}

Elizabeth A. Cudney, Kioumars Paryani, Kenneth M. Ragsdell. Applying the Mahalanobis-Taguchi System to Vehicle Handling. Concurrent Engineering: Research and Applications, 2006, 14 (4), pp.343354. 10.1177/1063293X06073568 . hal-00571200

\section{HAL Id: hal-00571200 https://hal.science/hal-00571200}

Submitted on 1 Mar 2011

HAL is a multi-disciplinary open access archive for the deposit and dissemination of scientific research documents, whether they are published or not. The documents may come from teaching and research institutions in France or abroad, or from public or private research centers.
L'archive ouverte pluridisciplinaire HAL, est destinée au dépôt et à la diffusion de documents scientifiques de niveau recherche, publiés ou non, émanant des établissements d'enseignement et de recherche français ou étrangers, des laboratoires publics ou privés. 


\title{
CONCURRENT ENGINEERING: Research and Applications
}

\section{Applying the Mahalanobis-Taguchi System to Vehicle Handling}

\author{
Elizabeth A. Cudney, ${ }^{1, *}$ Kioumars Paryani ${ }^{2}$ and Kenneth M. Ragsdell ${ }^{1}$ \\ ${ }^{1}$ University of Missouri - Rolla, UMR Design Engineering Center, Rolla, MO 65409, USA \\ ${ }^{2}$ General Motors Corporation, 30500 Mound Road, Warren, MI 48090, USA
}

\begin{abstract}
The Mahalanobis-Taguchi system (MTS) is a diagnosis and forecasting method using multivariate data. Mahalanobis distance (MD) is a measure based on correlations between the variables and patterns that can be identified and analyzed with respect to a base or reference group. The MTS is of interest because of its reported accuracy in forecasting using small, correlated data sets. This is the type of data that is encountered with consumer vehicle ratings. MTS enables a reduction in dimensionality and the ability to develop a scale based on MD values. MTS identifies a set of useful variables from the complete data set with equivalent correlation and considerably less time and data. This article presents the application of the MTS, its applicability in identifying a reduced set of useful variables in multidimensional systems, and a comparison of results with those obtained from a standard statistical approach to the problem.
\end{abstract}

Key Words: Mahalanobis-Taguchi system, multivariate, pattern recognition, orthogonalization, diagnosis, forecasting, multivariate.

\section{Introduction}

The primary objective of this research is to develop a methodology, which demonstrates the relationship between actions of the producer and suppliers (for instance, target setting of sub-systems or components' performance attributes) and consumer satisfaction ratings. The goal is to efficiently forecast consumer satisfaction ratings as a function of available vehicle level performance data for vehicle handling.

The purpose of this research is to develop a relationship between vehicle attributes and measured customer satisfaction ratings for the purpose of understanding and improving customer-driven quality. The Mahalanobis-Taguchi system (MTS) enables a reduction in dimensionality and the ability to develop a scale based on MD values [1].

Consumers measure quality and performance at the vehicle level, but important cost-effective decisions at the subsystem or component level must be made by the producer in order to satisfy, economically, consumer needs by providing affordable and high quality products. Consumers evaluate vehicle level attributes such as ride, handling, acceleration, braking, and roominess. These attributes are influenced by many factors at all levels of the vehicle architecture, and these factors are often correlated. The design problem would

*Author to whom correspondence should be addressed. E-mail: elizabeth cudney@umr.edu

Figures 1, 3, and 5-12 appear in color online: http://cer.sagepub.com be significantly simplified if vehicle attributes depended on a unique set of vehicle factors. That is, if one could isolate a set of factors for ride, another for handling, and yet others for, acceleration, roominess, etc; then the design of high quality, low cost vehicles would be much easier. Unfortunately, this is not the case. The situation is much more complex, because factors at the subsystem and part levels may affect several vehicle attributes, and for a single vehicle attribute the controlling factors are often correlated. An effective methodology is needed to handle this more realistic and complex situation. The methodology proposed will use the pattern recognition scheme known as the MTS to translate the performance of lower level elements into an estimate of consumer satisfaction rating at the vehicle level [2].

\section{Review of Relevant Literature}

Considerable research is available utilizing Mahalanobis distance for determining the similarities of values from known and unknown samples. However, existing research uses Mahalanobis distance (MD) to consider measurable and distinct variables. Consumer requirements or inputs to the research were concrete and clearly defined. However, this research will create a methodology to translate lower level characteristics using MD into performance specifications and capabilities.

Mahalanobis distance is utilized to determine the misclassification of samples in research by Shen et al. [3]. Two processes for tablet production 
are evaluated in this research including wet granulation and direct compression which are the two main methods used in tablet preparation. Pyrolysis-gas chromatography-mass spectrometry was used to discriminate the two processes. First, samples were removed that did not contain at least half the number of samples for one of the two classes. Principal components analysis (PCA) was then employed to determine the main principal components. Based on the PCA analysis, three factors and one sample were excluded. The data was further processed using Fisher discriminant analysis to classify the sample using unsupervised and supervised classification methods. Fisher discriminant analysis is an approach that is commonly used for feature extraction between groups [4]. The results were evaluated using MD to determine the misclassification rate.

Taguchi [5] utilized the MTS for diagnosis and pattern recognition. His research discussed a case study involving liver disease diagnosis in Tokyo, Japan using 15 variables. The normal group consisted of 200 people that were determined not to have liver disease. For each test group, he determined which variables were significant. In his research, Taguchi developed an eight-step procedure titled 'Mahalanobis Distance for Diagnosis and Pattern Recognition System Optimization Procedure'.

Garcia-Lagos et al. [6] utilized MD in a topology assessment for power systems. The research developed system architecture for use in a state estimator which worked with a bus-branch oriented network model. The architecture was developed in two stages including a preprocessing stage and a classification stage. The preprocessing stage transformed the measurements into output vectors for grid topologies. In the second stage, classification is determined using a layer of Gaussian potential function units based on the MD. The units are established through input from the preprocessed vector. This indicates the degree to which a vector belongs to a specific topology. This assessment enabled the researchers to identify the actual topology.

Jugulum and Monplaisir [7] performed preliminary comparison between MTS and neural networks using medical data with 15 variables. They compared both methods for large and small samples. The small sample was selected from the whole sample of 200 observations in the healthy group. They showed that in the case of large samples both methods perform equally well and in the case of small samples, MTS is somewhat better than neural networks. Jugulum did not compare these methods in terms of reducing the number of attributes.

Al-Otum [8] proposes two algorithms for color morphology. The first is a Mahalanobis-color-distance (MCD)-based morphological ordering algorithm. The second is a corrected component-wise morphological ordering algorithm. Both algorithms employ a Mahalanobis color measure based on reduced and conditional ordering of the data to perform four basic morphological operators: dilation, erosion, opening, and closing. The data ordering is performed using the MCD in which the angle-valued pixels are replaced by a scalar. The two algorithms were evaluated using a perceptual image quality assessment.

A comparison of the MTS and neural network was also provided in the work of Hong et al. [9]. Hong utilized a breast cancer study to compare the ability of MTS and neural network algorithm with varying numbers of attributes and different numbers of data size. The results indicated that MTS performed better with small sample sizes than neural network.

Mahalanobis distance was used to maximize productivity in a new manufacturing control system by Hayashi et al. [10]. The research used MD as a core to their manufacturing control system because of the method's ability to recognize patterns. Due to a very high utilization of certain processes, one of the manufacturing areas for wafers was experiencing a bottleneck. Approximately $50 \%$ of the primary tools in this manufacturing area had over $90 \%$ utilization. Mahalanobis distance was used in conjunction with manufacturing administrators to focus on routes that involve the shortest cycle time and reduced labor costs. The new system detected deviations from normal productivity much earlier, enabled root cause identification, and prioritized resolution.

Asada [11] used the MTS to forecast the yield of wafers. Yield of wafers is determined by the variability of electrical characteristics and dust. The research focused on one wafer product with a high yield. Mahalanobis distances were calculated on various wafers to compare the relationship between yield and distance [11]. The signal-to-noise ratios were used to indicate the capability of forecasting and the effect of the parameters.

A method for handling uncertainty in feature position is proposed in the work of Anandan and Irani [12]. The uncertainty in feature position for 3D shape and motion in multiple views depends on the underlying spatial intensity structure. The purpose of this research is to minimize the covariance-weighted squared error which is the MD. The raw data is transformed into a covariance-weighted space in which the noise is uncorrelated and equally distributed. Singular Value Decomposition (SVD) is then employed to minimize the objective function in the transformed data space. A second step is then performed to recover the shape and motion of the original data space. Results are provided for varying degrees of uncertainty.

Pattern recognition using MD was demonstrated in the work of $\mathrm{Wu}$ [13]. Wu used pattern recognition to diagnose human health. The results of tests from 
a regular physical check-up were used as the characteristics. The correlation between different tests was shown. Mahalanobis distance was used to summarize the multidimensional characteristics into one scale. In this research the base point was difficult to define because it was a healthy person. People who were judged to be healthy for the past 2 years were considered to be healthy and defined the normal group. The research considered diagnosis of liver function with the objective of forecasting serious disease until the next check-up. The approach provided a more efficient method and also avoided inhuman treatment which was previously used in double blind tests.

The research presented in this article is distinct from existing literature due to the nature of value perception. The focus is on consumer satisfaction which tends to be subjective rather than objective. Using consumer satisfaction ratings and vehicle performance data, from among a given set of variables the useful variables are determined. We then take MTS one step further to forecast consumer satisfaction ratings using regression analysis. Based on the regression equations, the impact on consumer satisfaction from changes in lower level components can be determined.

\section{Multicollinearity}

A system, by its nature, is multidimensional. In order to improve or optimize a system, it is necessary to understand the system variables and noise conditions that affect the system's performance. All variables do not affect the system's performance to an equal degree. Therefore, it is paramount to identify the critical set of variables through discriminant analysis of the system. The critical set of variables can then be used for diagnosis and prediction.

Generally speaking, a multidimensional system consists of an input signal, $M$, that is typically given by the consumer; noise conditions, that arise from changes in consumer use of the product, wear and deterioration, and manufacturing variation. The control factors are used to control performance of the system. The control factor levels are adjusted to provide, consistently, the required relationship between the system signal (input) and performance measure (output). A graphical depiction of a generalized multidimensional system is given in Figure 1.

An issue that must be addressed during the course of MTS analysis is multicollinearity. Multicollinearity occurs when there are strong correlations among two or more factors. Under this condition, the determinant of the correlation matrix will approach zero [14]. The resulting inverse matrix and scaled MD will be difficult to compute and are inaccurate if standard approaches are employed. We consider two methods to mitigate the

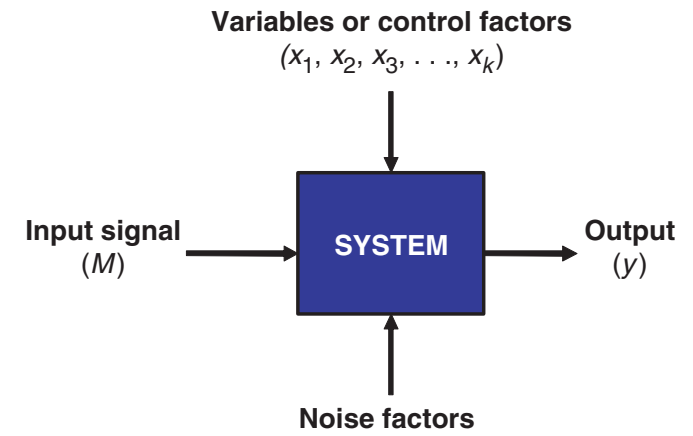

Figure 1. Multidimensional system.

effects of multicollinearity in MTS: the adjoint matrix (MDA) and the Gram-Schmidt orthogonalization.

\section{Mahalanobis Distance}

Prasanta Chandra Mahalanobis introduced MD in 1936 [1]. The MTS was later developed by Genichi Taguchi as a diagnosis and forecasting method using multivariate data for robust engineering.

Mahalanobis distance is a distance measure that is based on correlations between variables and the different patterns that can be identified and analyzed with respect to a reference population. $\mathrm{MD}$ is a discriminant analysis approach, which will be used to predict changes in consumer satisfaction corresponding to changes in multiple engineering characteristics at all levels of a hardware set (vehicle design) [1]. MD is different from Euclidean distance because it addresses the correlations and distribution of the data. Traditionally, the MD methodology has been used to classify observations into different groups. $\mathrm{MD}$ is defined in Equation (1).

$$
\mathrm{MD}_{j}=D_{j}^{2}=\frac{1}{k} Z_{i j}^{T} A^{-1} Z_{i j}
$$

where, $k$ is the total number of variables; $i$ the number of variables $(i=1,2, \ldots, k) ; j$ the number of samples $(j=1,2, \ldots, n) ; Z_{i j}$ the standardized vector of normalized characteristics of $x_{i j} ; x_{i j}$ the value of the $i$-th characteristic in the $j$-th observation; $m_{i}$ the mean of the $i$-th characteristic; $s_{i}$ the standard deviation of the $i$-th characteristic; $T$ the transpose of the vector; $A^{-1}$ the inverse of the correlation matrix.

Mahalanobis distance is used to determine the similarity of a known set of values (normal group) to that of an unknown set of values (abnormal group). It has been successfully applied to a broad range of cases mainly because it is very sensitive to inter-variable changes in data. Also, because the MD is measured in terms of standard deviations from the mean of the 
samples, it provides a statistical measure of how well an unknown sample matches a known sample set.

\section{The Mahalanobis-Taguchi System}

The MTS is a pattern recognition technology that aids in quantitative decision making by constructing a multivariate measurement scale using a data analytic method. The main objective of MTS is to make accurate predictions in multidimensional systems by constructing a measurement scale [1]. The pattern of observations in a multidimensional system depends on the correlation structure of the variables in the system. It is impossible to make intelligent decisions if the variables are considered one at a time. The variables that have the greatest impact on performance must be identified and used in concert. To construct a multidimensional measurement scale, it is important to have a distance measure. The distance measure is based on the correlation between the variables and the different patterns that are identified and analyzed with respect to a base or reference point.

In the MTS, the Mahalanobis space (MS), reference group, is obtained using the standardized variables of healthy or normal data. The Ms can then be used to discriminate between normal and abnormal objects. Once the MS is established, the number of attributes is reduced using an appropriate orthogonal array (OA) and signal-to-noise ratio (SN) by evaluating the contribution, or gain, of each attribute. Each row of the OA determines a subset of the original system by including and excluding that attribute to and from the system.

\subsection{Adjoint Matrix Approach}

In MTS analysis the adjoint of the correlation matrix may be used instead of the inverse matrix to calculate the distances to address the issue of multicollinearity. This approach can be used even when collinearity is not present. The adjoint of a square matrix $A$ is obtained by replacing each element of $A$ with its own cofactor and transposing the result. The symbol $\mathrm{MD}_{\mathrm{A}}$ is used to denote the MD obtained from an adjoint matrix. The relationship between the adjoint, determinant, and inverse is shown in the following equations:

$$
\begin{aligned}
& \mathrm{MD}_{\mathrm{A}}=\frac{1}{k} Z_{i j}^{T} C^{-1} Z_{i j} \\
& \mathrm{MD}_{\mathrm{A} j}=\frac{1}{k} Z_{i j}^{T} C_{\mathrm{adj}} Z_{i j}
\end{aligned}
$$

$$
\mathrm{MD}_{j}=\frac{1}{\operatorname{det} \cdot C} \mathrm{MD}_{\mathrm{A} j} .
$$

$\mathrm{MD}_{\mathrm{A}}$ is similar to $\mathrm{MD}$; however, there exist some important differences. MD and $\mathrm{MD}_{\mathrm{A}}$ both represent the distances from the healthy or normal group and can be used to measure the degree of abnormality. In the adjoint matrix approach, the MS also contains means, standard deviations, and the correlation structure of the healthy or normal group. However, here the MS is not called the unit space because the average of the $\mathrm{MD}_{\mathrm{A}}$ 's is not unity.

\subsection{Mahalanobis-Taguchi Gram-Schmidt}

Gram-Schmidt is an orthogonalization process which can be used to enhance the calculation of MDs when collinearity is present [1]. Mahalanobis-Taguchi Gram-Schmidt (MTGS) accounts for abnormal conditions that are extremely good by identifying the direction of abnormalities. Identifying the direction of abnormalities enables the distinction between 'good' or 'bad' abnormalities which increases the effectiveness of the forecasting process. This distinction cannot be made in the MTS method which uses the inverse of the correlation matrix. Identifying the direction of abnormals in multivariate systems is important so that the decision maker can treat the abnormally good group separately. For example, a vehicle that falls outside of the normal group but is rated high in the corresponding attribute by consumers should be regarded separately from vehicles in the abnormal group that are rated low.

MTGS identifies the useful variables based on orthogonal Gram-Schmidt vectors that allow the effects of the variables to be determined independently. Orthogonal arrays (OAs) must be used in MTGS if the effects of partial correlations are significant and a definite order for the variables cannot be defined. If correlation effects are not significant and a definite order of the variables exists, then MTGS is much simpler because an OA does not need to be used. MTGS provides guidelines to identify the direction of abnormals; however, there is still considerable discretion for the decision maker to determine the type of abnormal conditions.

The Gram-Schmidt process states that given linearly independent vectors $Z_{1}, Z_{2}, \ldots, Z_{k}$, there exist mutually orthogonal vectors $U_{1}, U_{2}, \ldots, U_{k}$ with the same span. Gram-Schmidt vectors are constructed using the standardized values of the variables. The standardized values are obtained by subtracting the mean from the original 


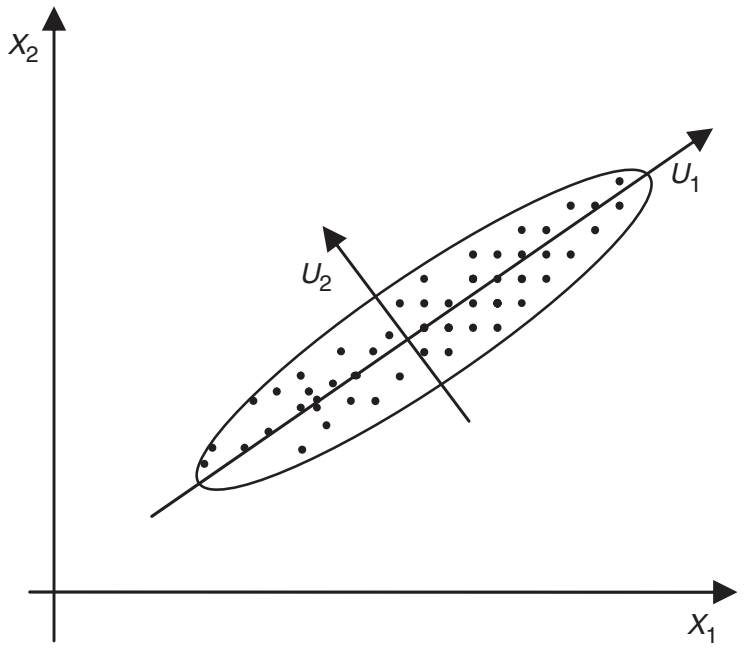

Figure 2. Gram-Schmidt vectors in two dimensions.

variable and dividing by the standard deviation. The Gram-Schmidt vectors are defined as follows:

$$
\begin{aligned}
U_{1}= & Z_{1} \\
U_{2}= & Z_{2}-\frac{Z_{2}^{T} U_{1}}{U_{1}^{\prime} U_{1}} U_{1} \\
\vdots & \\
U_{k}= & Z_{k}-\frac{Z_{k}^{T} U_{1}}{U_{1}^{T} U_{1}} U_{1} \\
& \quad \cdots-\frac{Z_{k}^{T} U_{k-1}}{U_{k-1}^{T} U_{k-1}} U_{k-1} .
\end{aligned}
$$

After performing the Gram-Schmidt process, we have the orthogonal vectors $U_{1}, U_{2}, \ldots, U_{k}$. Data points can be expressed as a function of the primal variables $x_{i}$ or as functions of the new spanning set $U_{1}, U_{2}$, etc. Figure 2 illustrates a $2 \mathrm{D}$ graphical representation of the data points using the Gram-Schmidt orthogonalization.

\section{Vehicle Handling}

The MTS can be used to minimize the number of variables required to forecast the performance of a system. The steps of the MTS analysis for vehicle handling are outlined in this section. The objective of the MTS analysis for vehicle handling is to explain the relationship between vehicle handling parameters and measured customer satisfaction rating. The purpose is to provide an understanding of the relationship and offer an opportunity to improve, efficiently, customer-driven quality.

\subsection{Outlier Detection}

Any data set contains outliers. Outliers are data points or observations that are not consistent with the primary trend of the data and may cloud the analysis results. The decision was made to identify outliers and remove them from the data set prior to MTS analysis.

The first step in the MTS analysis of the handling metrics was to remove the outliers using MD. Outliers may not be easily identified using the data in the primal space. Using MTS, we are able to look at data in a transformed space, the MS. This allows us to identify trends. Outliers become clear using the MS. The MD was calculated for all of the 72 data points provided using the 21 vehicle handling parameters (Figure 3).

Using a threshold value for MD of 1.5, four data sets (i.e., vehicles) were determined as outliers, i.e., 7, 36, 38, and 44. The outliers were removed prior to running the MTS analysis using MDA and MTGS. In MTS/MTGS, the threshold is essentially a safety factor. The threshold is typically used in MTS during the final stage to monitor conditions using the measurement scale. In this research, a breakthrough procedure for identifying outliers using MTS and a threshold value was developed. Observations (values for certain vehicles attribute) above the threshold value are classified as outliers. The threshold is used for the purpose of identifying outliers because it determines the general condition of the data. Theoretically, the threshold is determined using the quality loss function (QLF). In MTS, the concept of QLF can be extended to determine the thresholds in multivariate diagnosis. The QLF determines the threshold as the balance point between the cost of including a factor and the cost of not including it. The QLF equation is given in Equation (8) [9].

$$
\text { Loss }=\frac{A_{0}}{\Delta_{0}^{2}} \mathrm{MD}
$$

where, $A_{0}$ is the loss associated with $\Delta_{0}$, and $\Delta_{0}$ is the functional limit.

The QLF associates a dollar value to the quality of a product or process. The measured loss is estimated using Equation (8). A graphical representation of the QLF is given in Figure 4 [15].

The threshold value is utilized prior to MTS analysis to identify outliers. However, the threshold is ordinarily utilized in the fourth stage of MTS which involves future diagnosis using the useful variables. In the fourth stage, the threshold is used while monitoring the conditions to make decisions. The threshold has a strong impact on the accuracy of diagnosis. Incorrect thresholds may cause inaccurate decisions which result in the loss. It is important to select the proper threshold to minimize the total loss. In the event that the data is not available for consumer loss, consumer tolerance, etc., the threshold decision is judgmental and using a visual cut off point is customary. 


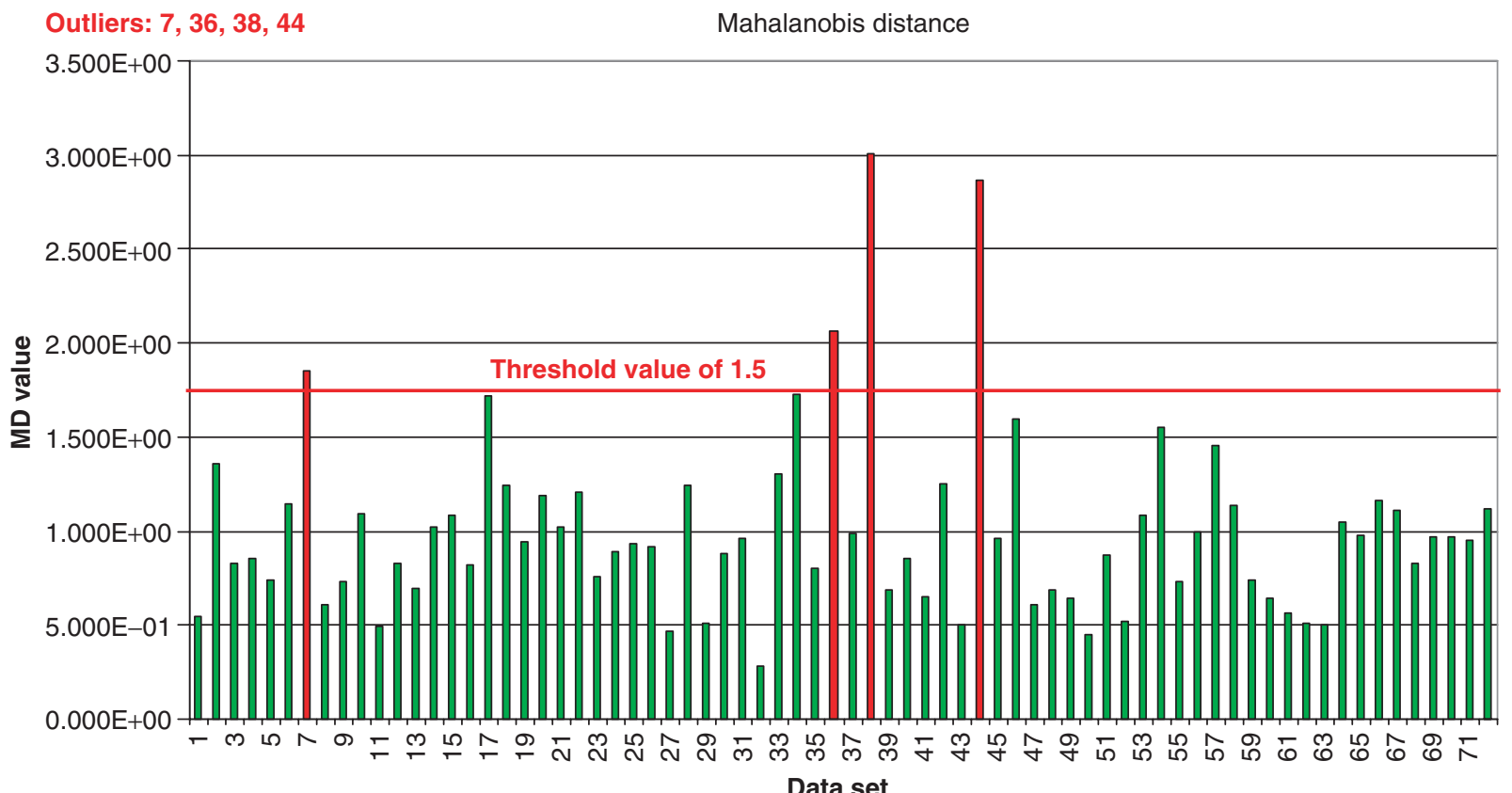

Figure 3. MD values for handling metrics.

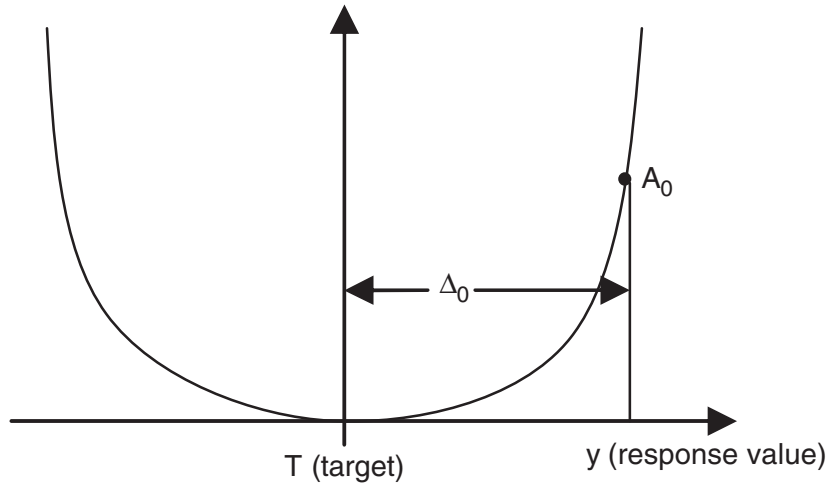

Figure 4. Quality loss function.

\subsection{Vehicle Handling Analysis Using the Adjoint Matrix}

The remaining 68 samples were used to perform the handling analysis using the adjoint matrix. The 21 original vehicle handling parameters were used in the analysis. The first step in calculating $\mathrm{MD}_{\mathrm{A}}$ is to define a 'normal' or 'healthy' group as the MS. Twenty-seven samples were selected for the normal group based on their customer satisfaction ratings. Six samples that fell outside of the normal group were selected as the test group. The MD was then calculated for the normal group. Table 1 shows the resulting MD value for each sample from the normal group. The MDs were then calculated for the test group. Table 2 summarizes these MDs. A summary of the MD values for the normal group and test group is provided in Figure 5.
Table 1. Normal group $M D_{A}$ values.

\begin{tabular}{lc}
\hline Dataset & MD \\
\hline 4 & 2.33381 \\
39 & 2.40739 \\
14 & 2.25754 \\
6 & 2.31645 \\
50 & 1.75794 \\
64 & 2.58338 \\
66 & 2.20773 \\
43 & 1.72969 \\
71 & 2.46424 \\
69 & 2.43677 \\
65 & 2.32682 \\
24 & 2.23266 \\
62 & 1.86937 \\
26 & 2.09679 \\
22 & 2.22263 \\
41 & 1.58935 \\
60 & 2.39574 \\
25 & 1.81629 \\
27 & 1.99299 \\
32 & 0.922596 \\
33 & 2.49108 \\
49 & 2.17796 \\
9 & 2.37491 \\
29 & 1.86593 \\
28 & 2.38188 \\
18 & 2.50826 \\
20 & 2.53231 \\
\hline
\end{tabular}

The next step is to optimize the system. An L32 orthogonal array was used for the optimization [16]. The useful set of variables is obtained by evaluating the gain, measured in decibels, associated with each factor. 
Table 2. Test group $M D_{A}$ values.

\begin{tabular}{lc}
\hline Dataset & MD \\
\hline 47 & 12.7102 \\
53 & 29.1724 \\
55 & 8.2042 \\
51 & 6.63564 \\
52 & 6.53469 \\
31 & 7.57257 \\
\hline
\end{tabular}

Gain is the average difference in the magnitudes of signals using the $\mathrm{S} / \mathrm{N}$ ratio measured in decibels when a variable is excluded and included. The gains for the 21 handling metrics are shown in Figure 6, 14 of which are useful.

Regression analysis is then performed. The useful variables are used in the regression analysis. Using the regression equation for the 14 factors determined to be useful variables, the predicted customer satisfaction ratings are calculated. The predicted ratings are compared to the actual customer satisfaction ratings graphically. Scatter plots were then developed to show

Bar graph normal group vs. test group

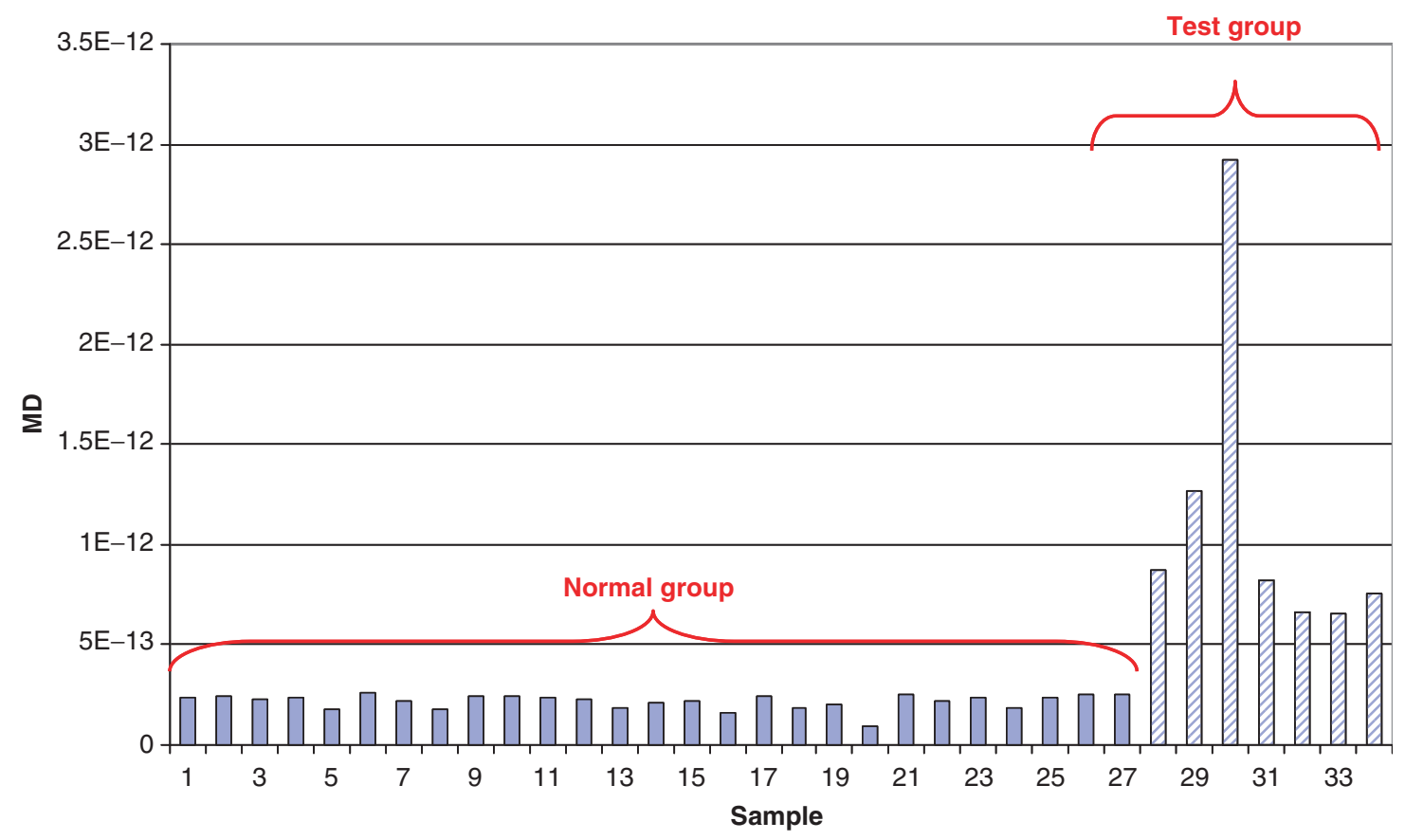

Figure 5. MDs for normal group vs test group.

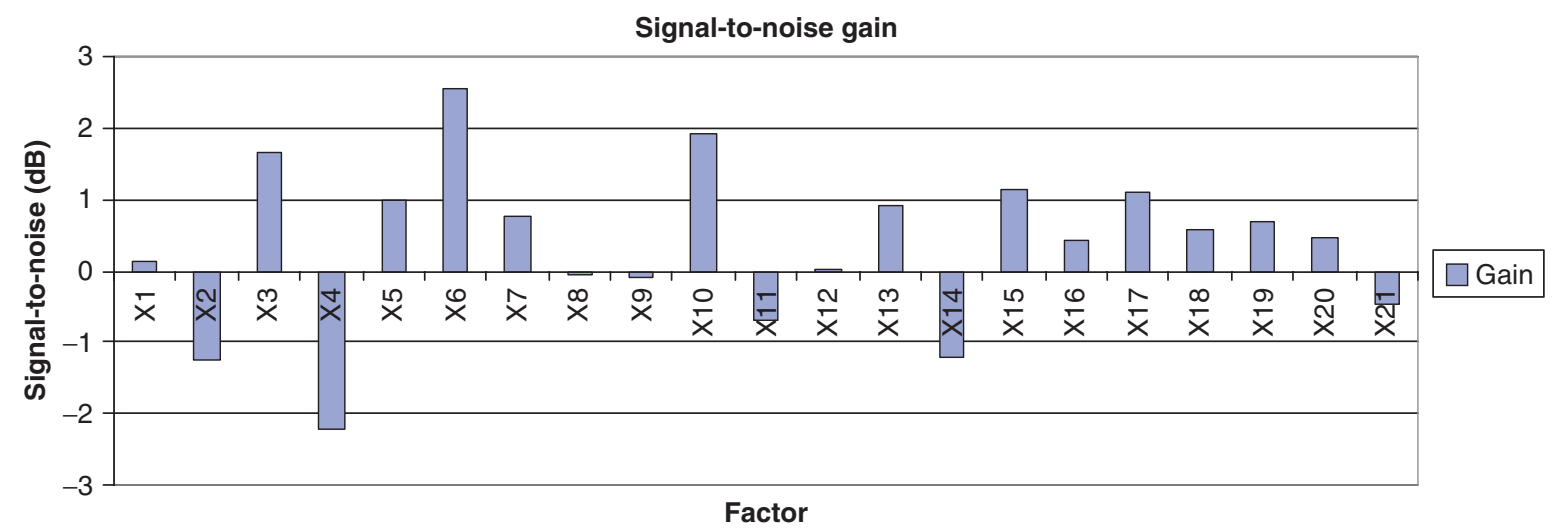

Figure 6. Handling index gains. 


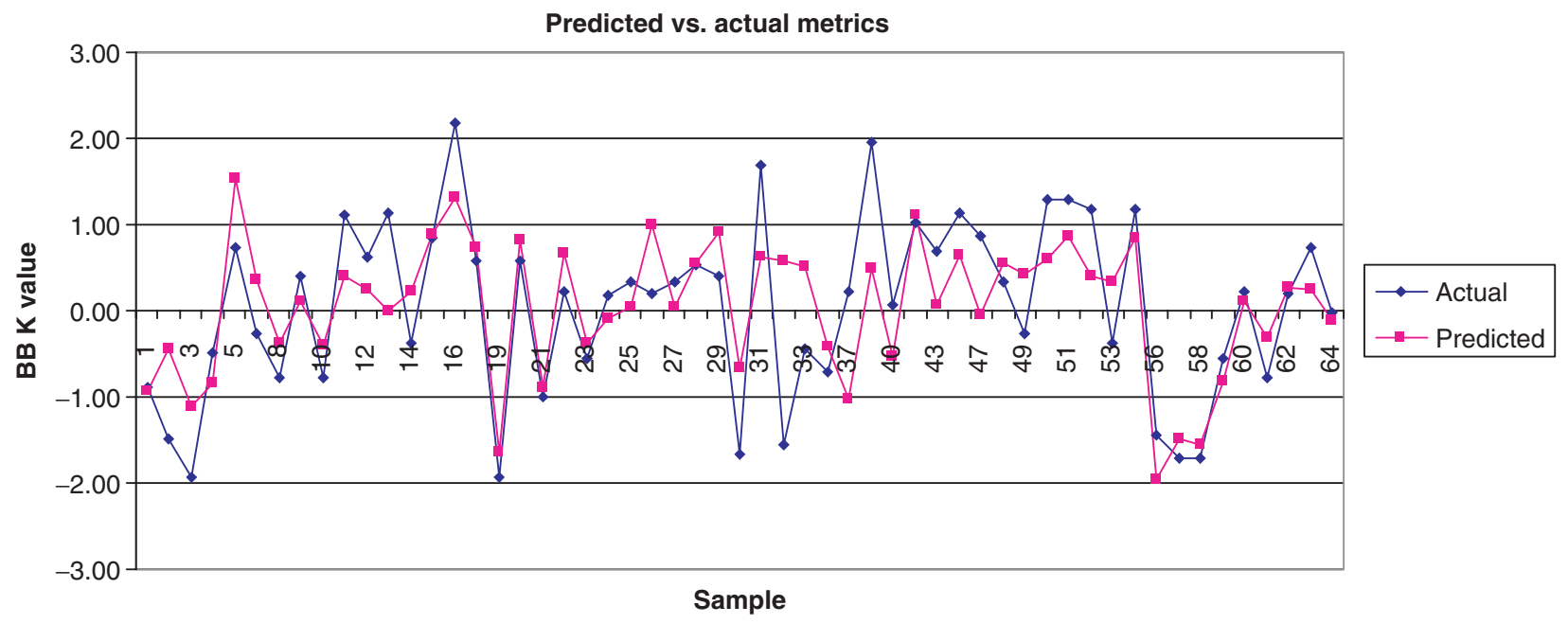

Figure 7. Measured vs predicted customer satisfaction ratings for the 14 useful variables.

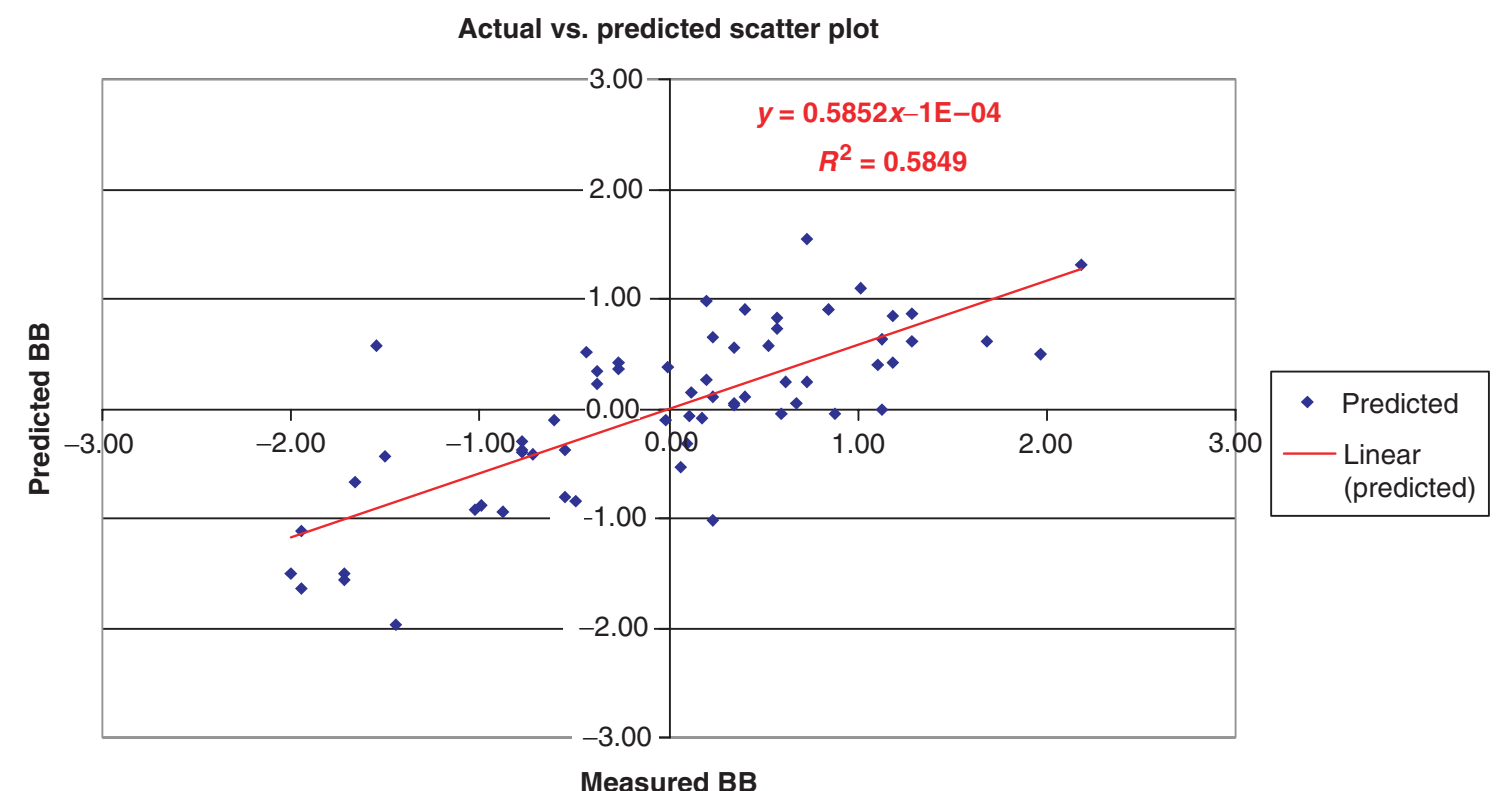

Figure 8. Scatter plot for measured vs predicted customer satisfaction ratings for the 14 useful variables.

the correlation between the measured versus predicted customer satisfaction ratings (Figures 7 and 8).

\subsection{Handling Metrics Analysis Using Mahalanobis-Taguchi Gram-Schmidt Method}

The remaining 68 samples (after removing outliers) were also used to perform the handling analysis using the MTGS method. The 21 original vehicle handling parameters were used in the analysis.

The first step in calculating $\mathrm{MD}_{\mathrm{GS}}$ is to define a 'normal' or 'healthy' group as the MS. The MD values were calculated for the reference group. The results are shown in Table 3. The MD values were then calculated for the test group. The results are shown in Table 4. A bar graph illustrating the difference in MD values for the normal and test groups is shown in Figure 9.

The next step is to optimize the system using an L32 orthogonal array and dynamic signal-to-noise ratio. The useful set of variables is obtained by evaluating the gain in decibels associated with each variable. The gains for the 21 vehicle handling parameters are shown in Figure 10.

Regression analysis is then performed by adding the 15 useful variables. Using the regression equation, the predicted customer satisfaction rating is calculated. A graph illustrating the measured and predicted customer satisfaction ratings is given in Figure 11. A scatter plot was then developed to show the 
Table 3. Reference group $M D_{G s}$ values.

\begin{tabular}{lc}
\hline Dataset & MD \\
\hline 4 & 1.041 \\
39 & 1.074 \\
14 & 1.007 \\
6 & 1.033 \\
50 & 0.784 \\
64 & 1.152 \\
66 & 0.985 \\
43 & 0.771 \\
71 & 1.099 \\
69 & 1.087 \\
65 & 1.038 \\
24 & 0.996 \\
62 & 0.834 \\
26 & 0.935 \\
22 & 0.991 \\
41 & 0.709 \\
60 & 1.069 \\
25 & 0.810 \\
27 & 0.889 \\
32 & 0.412 \\
33 & 1.111 \\
49 & 0.971 \\
9 & 1.059 \\
29 & 0.832 \\
28 & 1.062 \\
18 & 1.119 \\
20 & 1.129 \\
\hline
\end{tabular}

Table 4. Test group $M D_{G s}$ values.

\begin{tabular}{lr}
\hline Dataset & MD \\
\hline 52 & 2.807 \\
51 & 2.850 \\
31 & 3.252 \\
55 & 3.524 \\
47 & 5.459 \\
53 & 12.530 \\
\hline
\end{tabular}

correlation value between the actual and predicted ratings for customer satisfaction (Figure 12).

\section{Conclusions}

MTS enables a reduction in dimensionality and the ability to develop a scale based on MD values. MTS identifies a set of useful variables from the complete data set with equivalent correlation and considerably less time and data resulting in lower cost.

Twenty-one vehicle handling metrics were analyzed. Of the original 21 metrics, 14 were determined to be useful using the adjoint matrix method. Using MTGS, 15 metrics were determined to be useful. The correlation coefficient between the actual and predicted values is 0.765 and 0.891 for MDA and MTGS, respectively. MTGS identified an additional useful factor which increased the correlation coefficient. Handling experts preferred to use the 15 useful variables as identified by MTGS.

\section{Future Research}

Further research should be conducted to utilize the methodology developed in other contexts and industries that drive the requirements down throughout all levels of a system. The analysis performed relates subsystem levels to consumer satisfaction. Future research should incorporate subsystems, sub-subsystems, and components levels to fully gain the relationship between design changes made at these lower level components and overall consumer satisfaction. This will enable the producer to quantify the impact on consumer satisfaction for actions such as changing suppliers for a particular component or modifying design specification.

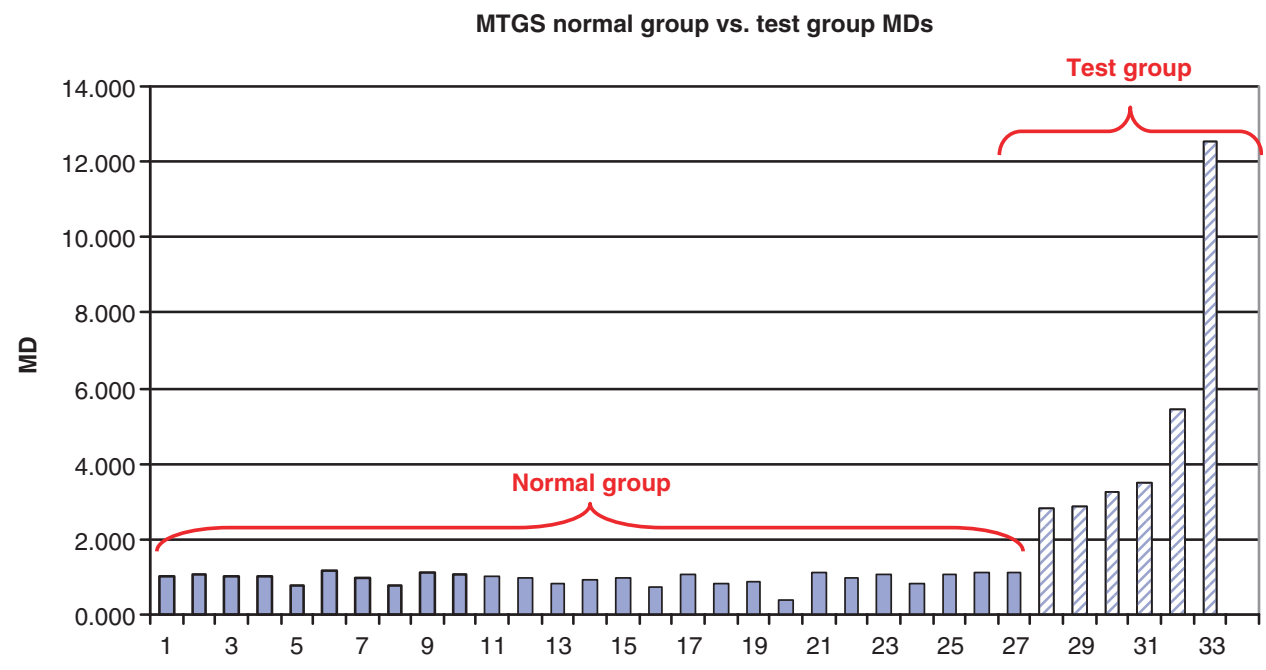

Figure 9. Normal group vs test group MD values. 


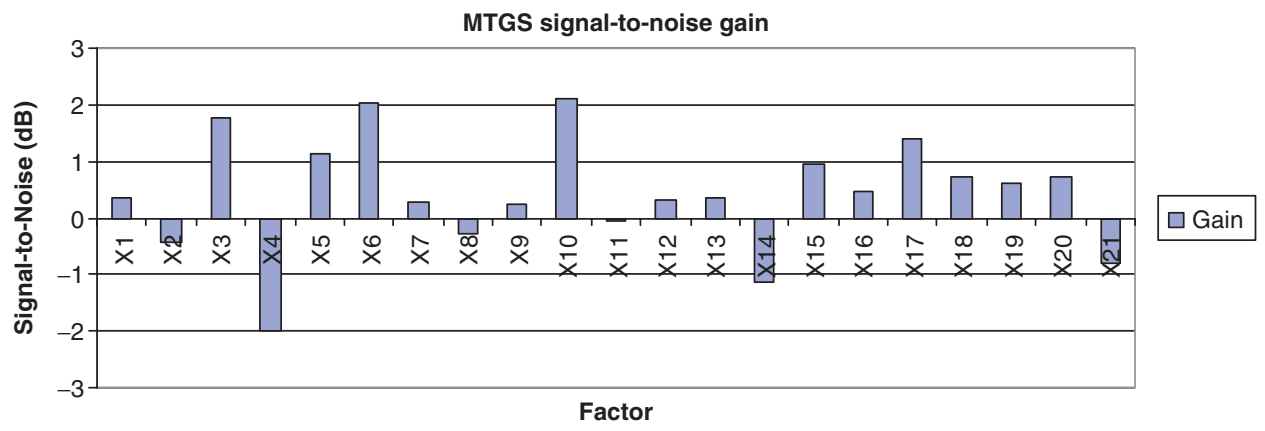

Figure 10. Gains.

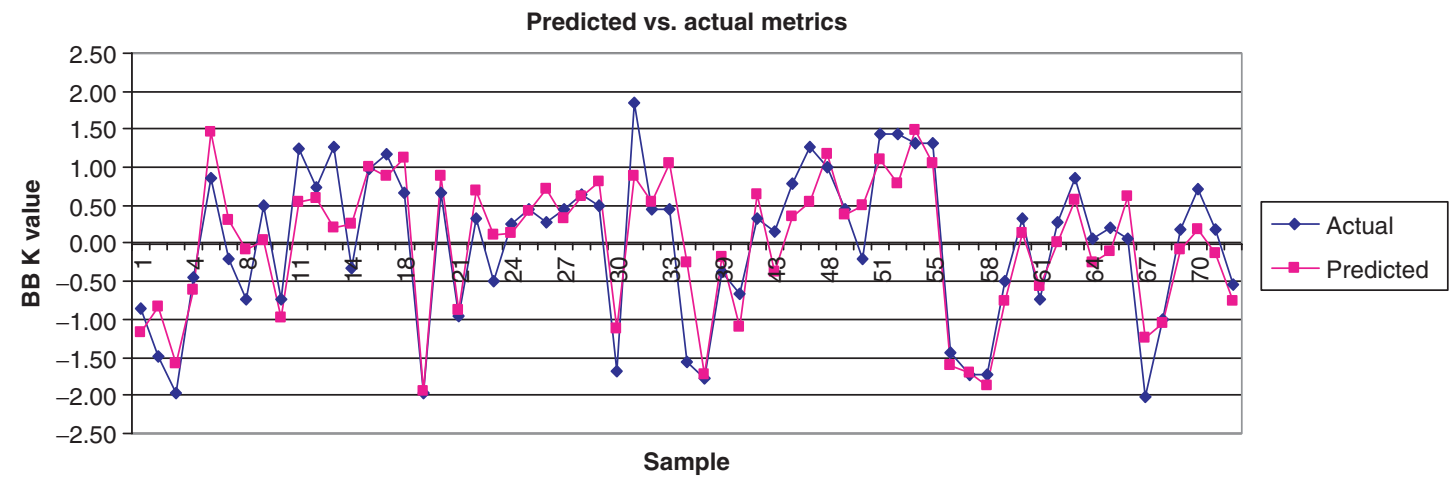

Figure 11. Measured vs predicted customer satisfaction ratings for the 12 useful variables.

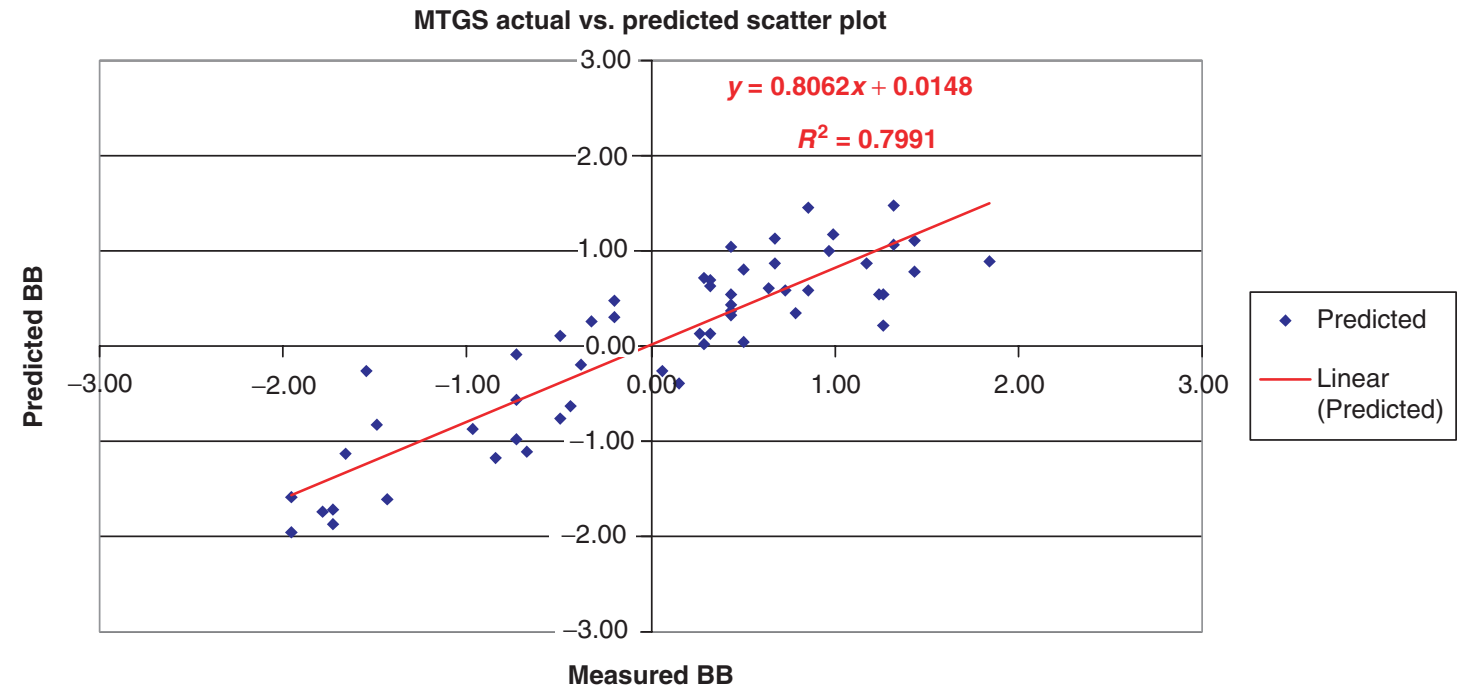

Figure 12. Scatter plot for measured vs predicted customer satisfaction ratings for the 15 useful variables.

The methodology proposed will provide information for making business decisions.

A key aspect that should be incorporated into this analysis is cost data. Important business decisions are made on the basis of cost and consumer impact. System optimization would be based on equations using cost data and constraints in the feasible region for components specifications. The objective function would be to minimize the cost and maximize consumer satisfaction.

\section{References}

1. Taguchi, G. and Jugulum, R. (2002). The MahalanobisTaguchi Strategy, New York: John Wiley \& Sons, Inc.

2. Cudney, E., Hong, J., Jikar, V., Paryani, K. and Ragsdell, K. (2005). Evaluation of the Effect of Vehicle Performance Attributes on Consumer Satisfaction: Progress Report 1, 1-38.

3. Shen, H., Carter, J.F., Brereton, R.G. and Eckers, C. (2003). Discrimination Between Tablet Production Methods Using Pyrolysis-Gas Chromatography-Mass 
Spectrometry and Pattern Recognition, Analyst, 128(3): 287-292.

4. Christensen, R. (1998). Analysis of Variance, Design and Regression: Applied Statistical Methods, New York: Chapman \& Hall/CRC.

5. Taguchi, S. (2000). Mahalanobis-Taguchi System, ASI Taguchi Symposium, 2000.

6. Garcia-Lagos, F., Joya, G., Marin, F.J. and Sandoval, F. (2003). Modular Power System Topology Assessment Using Gaussian Potential Functions, In: IEE Proceedings-Generation Transmission and Distribution, Vol. 150, No. 5, September 2003, pp. 635-640.

7. Jugulum, R. and Monplaisir, L. (2002). Comparison Between Mahalanobis-Taguchi- System and Artificial Neural Networks, Journal of Quality Engineering Society, 10(1): $60-73$.

8. Al-Otum, H.M. (2003). Morphological Operators for Color Image Processing Based on Mahalanobis Distance Measure, Optical Engineering, 42(9): 2595-2606.

9. Hong, J., Cudney, E.A., Taguchi, G., Jugulum, R., Paryani, K. and Ragsdell, K. (2005). A Comparison Study of Mahalanobis-Taguchi System and Neural Network for Multivariate Pattern Recognition, $A S M E$ IMECE Proceedings.

10. Hayashi, S., Tanaka, Y. and Kodama, E. (2001). A New Manufacturing Control System Using Mahalanobis Distance for Maximizing Productivity, IEEE Transactions, 14(4): 59-62.

11. Asada, M. (2001). Wafer Yield Prediction by the Mahalanobis-Taguchi System, IIE Transactions, 14(4): 25-28.

12. Anandan, P. and Irani, M. (2002). Factorization with Uncertainty, International Journal of Computer Vision, 49(2-3): 101-116.

13. Wu, Y. (1996). Pattern Recognition Using Mahalanobis Distance, TPD Symposium: 1-14.

14. Manly, B.F.J. (1994). Multivariate Statistical Methods: A Primer, London: Chapman \& Hall.

15. Kiemele, M.J., Schmidt, S.R. and Berdine, R.J. (1999). Basic Statistics: Tools for Continuous Improvement, Colorado Springs, CO: Air Academy Press, 8.6-8.7.

16. Fowlkes, W.Y. and Creveling, C.M. (1995). Engineering Methods for Robust Product Design, Reading, MA: Addison-Wesley, 371-381.

\section{Elizabeth A. Cudney}

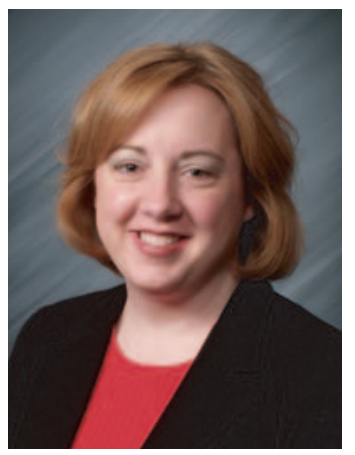

Elizabeth Cudney received her BS in Industrial Engineering from the North Carolina State University. She received her Master of Engineering in Mechanical Engineering with a Manufacturing Specialization and Master of Business Administration from the University of Hartford. She received her doctorate in Engineering Management from the University of Missouri - Rolla in May 2006. She is currently a Post Doctoral Fellow at the University of Missouri - Rolla.
Beth is an ASQ Certified Quality Engineer and Certified Six Sigma Black Belt. Beth is a member of the ASME, ASQ, IIE, SAE, SAVE, SME, and APICS. Beth's major areas of interest are in quality and lean manufacturing. She has published over 30 articles on topics including the Mahalanobis-Taguchi System, Robust Design, Lean manufacturing, and Six Sigma. She also coauthored two workshops for APICS on standard work and $5 \mathrm{~S}$ for their Lean Series Workshops.

\section{Kenneth M. Ragsdell}

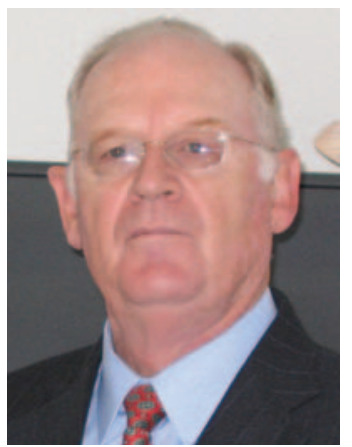

Professor Ragsdell received his BS and MS in Mechanical Engineering from the University of Missouri Rolla and his PhD from the University of Texas at Austin. Dr Ragsdell is a graduate of the General Motors Quality College, and a founding member of the Board of Directors of the Missouri Quality Award. He later served on the Board of Overseers of the Missouri Quality Award.

Dr Ragsdell's major area of expertise (in teaching, research, and practice) is the engineering design process with a specialty in computer-aided design, optimization, and quality engineering. $\mathrm{He}$ is the author of over 100 technical publications and a significant number of technical reports. Dr Ragsdell is a Fellow of the American Society of Mechanical Engineers, and has won the ASME Design Automation Award in 1993. Dr Ragsdell was elected a Senior Fellow of the Japan Society for the Promotion of Science and spent the month of December 1995 lecturing throughout Japan on Quality, Engineering and Management.

\section{Kioumars Paryani}

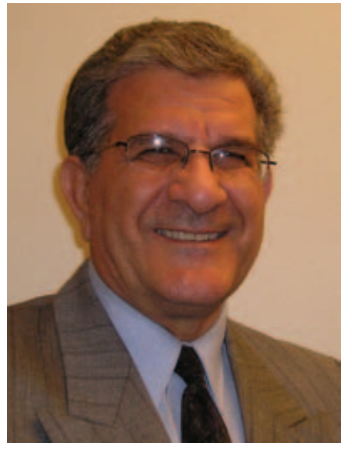

Kioumars Paryani received his BS in Electrical Engineering from Tehran Polytechnique with honors, and both his $\mathrm{MS}$ and $\mathrm{PhD}$ in Electrical Engineering from Michigan State University.

Prior to joining GM Research Laboratories in 1986, he spent 14 years teaching and doing research in the 
academia and research institutes in the areas of Industrial Engineering, Systems Engineering, and Operation Research. He started at GM Research Laboratories working on Project Trilby. His main responsibility with the Mission Analysis and Systems Development and Methodology groups was to do modeling and applying decision sciences and systems engineering. 